\title{
La fiabilité appliqué à la conception du dispositifs spectromètre mobile I ; optimisation des paramètres de vols
}

\section{The reliability applied to the design of the mobile spectrometer devices I; optimization of flight parameters}

\author{
Mohamed Abdessamia Chakchouk ${ }^{1,2}$, Abdelkhalak El Hami ${ }^{3}$, Mohamed Haddar ${ }^{4}$ \\ ${ }^{1}$ LMN, INSA de Rouen, Saint Etienne de Rouvray, 76800, France, mohamedabdelsami1992@gmail.com \\ ${ }^{2}$ Ecole Nationale des Ingénieurs de Sfax, Tunisie, mohamedabdelsami1992@gmail.com \\ ${ }^{3}$ LMN, INSA de Rouen, Saint Etienne de Rouvray, 76800, France, abdelkhalak.elhami@insa-rouen.fr \\ ${ }^{4}$ LA2MP, Ecole Nationale des Ingénieurs de Sfax, Tunisie, mohamed.haddar@enis.rnu.tn
}

RÉSUMÉ. Ce papier traite le problème de stabilisation de vol d'un drone équipé de capteur infrarouge dans un contexte de fiabilisation de la mesure.

Dans un premier temps on passe par une analyse de risque pour le spectromètre IR utilisé pour quantifié l'impact des vibrations sur le capteur, dans un second lieu la conception du dispositif capteur va être introduite pour déterminer la masse et le centre de gravité à l'aide de l'outil solid works, finalement les paramètre du contrôleurs de vol vont être optimisé à fin de trouver une stabilité optimale pour le drone lors de la phase de décollage.

ABSTRACT. The instructions put together below fall into four categories. The publisher would be grateful to authors for respecting these indications. The length of this summary may attain a dozen lines. It is to be written in size Arial 9 , spacing 13 points. An abstract in French will be joined.

MOTS-CLÉS. fiabilité, drone, spectrométrie IR, capteur infrarouge, paramètre de vol.

KEYWORDS. reliability, drone, IR spectrometry, infrared sensor, flight parameter.

\section{Introduction}

La simplicité de l'analyse spectrométrique, rapidité d'analyse et la grande sélectivité sont des raisons qui peuvent qualifier ces méthodes pour résoudre de nombreux problèmes de différents analytes. La relation entre concentration d'analyte dans le matériau ou l'échantillon et le signal qui a été enregistré est plutôt compliqué. En effet un signal d'analyte dépend généralement non seulement de la concentration d'analyte présente dans l'échantillon, mais de la concentration de toutes les autres substances, la structure du matériau, la forme de l'échantillon, Conditions environnementales.

De plus, la précision et la fiabilité de l'analyse chimique dépendent en grande partie de la façon dont l'échantillon analysé est préparé, car le mécanisme de préparation est spécifique dans les mesures chimiques.[1]

La méthode d'analyse spectrophotométrique est généralement groupe de méthodes de mesure utilisé dans les laboratoires d'analyse chimique, qu'on veut pousser dans ce projet vers des domaines d'utilisation plus dynamique, notamment les drones. Cependant, l'évaluation métrologique des résultats de la mesure photométrique sont insuffisants. 


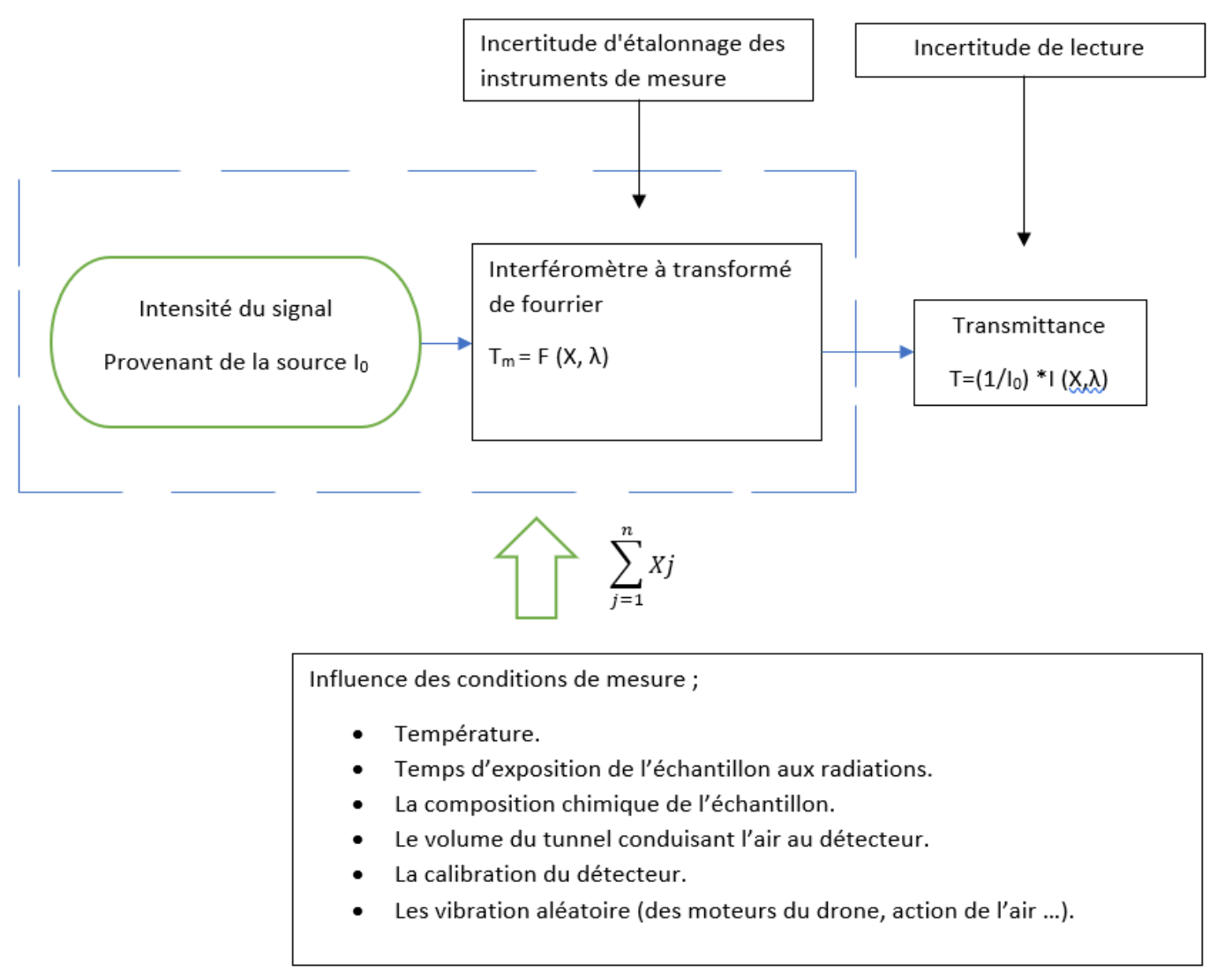

Figure 1. prise en compte des incertitudes

\section{Analyse de risques pour le capteur embarqué :}

Le diagramme ci-dessus montre que l'objet testé et la mesure l'instrument ne peut pas être séparé pendant l'évaluation du processus.

Selon le diagramme, afin d'évaluer minutieusement un résultat de mesure, tous les facteurs qui peuvent influencer les résultats de mesure doivent être analysés.

Ces facteurs sont :

-Température.

-Temps d'exposition de l'échantillon aux radiations.

-La composition chimique de l'échantillon.

-Le volume du tunnel conduisant l'air au détecteur.

-La calibration du détecteur.

-Les vibration aléatoire (des moteurs du drone, action de l'air ...).

En traitement. Ce n'est qu'après une analyse approfondie des facteurs susmentionnés et l'évaluation des conditions supplémentaires d'un certain cas, l'incertitude comme estimation précise du résultat de la mesure peut être correctement fourni.

Le tableau suivant montre l'impact de chaque paramètre sur la mesure pour un spectromètre IR. 


\begin{tabular}{|c|c|c|c|c|c|c|}
\hline $\begin{array}{c}\text { Méthodes } \\
\text { Spectrométrique }\end{array}$ & $\begin{array}{c}\text { Impact de } \\
\text { Impuretés } \\
\text { (Vapeur } \\
\text { d'eau, } \\
\text { aérosol) }\end{array}$ & $\begin{array}{c}\text { Temps } \\
\text { de } \\
\text { mesure }\end{array}$ & $\begin{array}{c}\text { Le volume dans } \\
\text { lequel se trouve } \\
\text { l'échantillon }\end{array}$ & $\begin{array}{c}\text { Température } \\
\text { D'échantillon }\end{array}$ & $\begin{array}{c}\text { La stabilité } \\
\text { du dispositif }\end{array}$ & Pression \\
\hline FTIR & + & + & + & + & + & \\
\hline
\end{tabular}

Tableau 1. analyse de risque

La fluctuation des charges et les incertitudes relatives aux modèles d'analyse contribuent à rendre la performance de la conception optimale différente de celle attendue. Une analyse de fiabilité peut être menée via les méthodes de simulation, couplées avec le modèle de masse et forces extérieures pour passer à une analyse de stabilité du drone.[2]

\section{Modélisation du vol vertical d'un drone et conception de son pilote automatique}

On considère le vol vertical d'un drone quadricoptère. Seule la dimension ' $z$ ' est prise en compte. Le drone est soumis à son poids ' $\mathrm{P}$ ' (on prend $\mathrm{g}=10$ ) et à la traction verticale de ses 4 hélices ' $\mathrm{T}$ '. Sa masse est ' $M$ '. Il subit de plus une force visqueuse aérodynamique proportionnelle et de sens inverse à sa vitesse verticale.

$$
\mathrm{F}=-\mathrm{Kv} * \mathrm{~V}
$$

- Equation différentielle du mouvement vertical ;

$$
\mathrm{M} \ddot{z}+\mathrm{K}_{\mathrm{v}} \dot{z}+\mathrm{M}^{*} \mathrm{~g}=\mathrm{T} \text {. }
$$

- Linéarisation autour de l'équilibre statique ;

Autour de la position d'équilibre on peut dire que l'action des ailette $\mathrm{T}$ tend vers le poids $\mathrm{P}$ et put s'écrire sous la forme ;

$$
\mathrm{T}=\mathrm{P}+\Delta \mathrm{T}=\mathrm{Mg}+\Delta \mathrm{T}
$$

Si on injecte cette égalité dans l'équation différentielles du mouvement on obtient une équation avec des termes qui dépende tous de $Z$ (mouvement verticale on prend seulement l'axe Oz), on peut dire que l'équation est linéarisé :

$$
\Delta \mathrm{T}=\mathrm{M} \ddot{z}+\mathrm{K}_{\mathrm{v}} \dot{Z}
$$

Ecrivons maintenant la fonction de transfert entre $\Delta \mathrm{T}$ et $\mathrm{Z}$ qui permettra après de concevoir un régulateur, il est ainsi important d'écrire la fonction de transfert sous la forme :

$$
\frac{A(P)}{1+A(p) * B(P)}
$$

$\Delta \mathrm{T}=\left(\mathrm{M}^{*} \mathrm{~s}^{2}+\mathrm{K}_{\mathrm{v}} * \mathrm{~s}\right) * \mathrm{Z}$

On sait que la fonction de transferts 'écrit sous la forme ; $\left(b_{m} * p^{m}+\ldots+b_{0}\right) /\left(a_{n} * p^{n}+\ldots+\right.$ $\left.a_{c}^{*} p^{c}\right)$

Donc pour déterminer l'ordre et le gain statique on a besoin de l'écrire sous la forme : 
$\left(1 / \mathrm{p}^{\mathrm{c}}\right) *\left(\mathrm{~b}_{0} / \mathrm{a}_{\mathrm{c}}\right) * \mathrm{H}(\mathrm{P})$ avec $\left(\mathrm{b}_{0} / \mathrm{a}_{\mathrm{c}}\right)$ le gain statique.

Pour notre fonction on a :

$$
\mathrm{Z}=\frac{1}{s} * \frac{1}{M s+K}
$$

On a $\mathrm{V}=\mathrm{s}^{*} \mathrm{z}$ (transformé de Laplace Vitesse de monté verticale) on peut dons déduire que le premier ordre donne la forme de $\mathrm{V}$.

On peut voir l'existence d'un pole nul au dénominateur, la condition de stabilité pour le système c'est si et seulement si ses pole de transmission appartiennent tous au demi-plan gauche donc tous strictement négatif, on peut dire que cette fonction de transfert est instable.

Revenons maintenant à la fonction du premier ordre qui donne la vitesse de monté $\mathrm{V}$ on remarque que le qain statique est : $1 / \mathrm{K}_{\mathrm{v}}$, la constante de temps est $\mathrm{M} / \mathrm{K}_{\mathrm{v}}$

Appliquons cette formule sur le cas du drone utilisé ;

$\left.\begin{array}{l}\mathrm{M}=5.33 \\ \mathrm{~K}_{\mathrm{v}}=0.02\end{array}\right\} \quad \mathrm{T}=266.5 \mathrm{~s}$

Voici ci-joint les effets que peut avoir un PID sur la stabilité du vol du drone [3] ;

\begin{tabular}{|c|c|c|c|}
\hline & trop bas & optimale & trop haut \\
\hline P & $\begin{array}{c}\text { Commande } \\
\text { lente }\end{array}$ & $\begin{array}{c}\text { Commande } \\
\text { réactives et } \\
\text { précises }\end{array}$ & Vibration \\
\hline I & $\begin{array}{c}\text { Difficulté à } \\
\text { maintenir la } \\
\text { position }\end{array}$ & Position stable & $\begin{array}{c}\text { Mouvement } \\
\text { instable }\end{array}$ \\
\hline D & Rebond & Arrêt net & $\begin{array}{c}\text { Chauffe } \\
\text { excessive des } \\
\text { moteurs }\end{array}$ \\
\hline
\end{tabular}

Tableau 2. impact des PID

On sait que pour un système de premier ordre $\mathrm{T}_{\mathrm{r}}=3 * \mathrm{~T}=799.5 \mathrm{~s}=13.25 \mathrm{~min}$

Le temps de réponse pour que la vitesse de monté se stabilise est de 13.25 min un temps assez long.

On peut aussi remarque le caractère instable par une simulation sur scilab : 


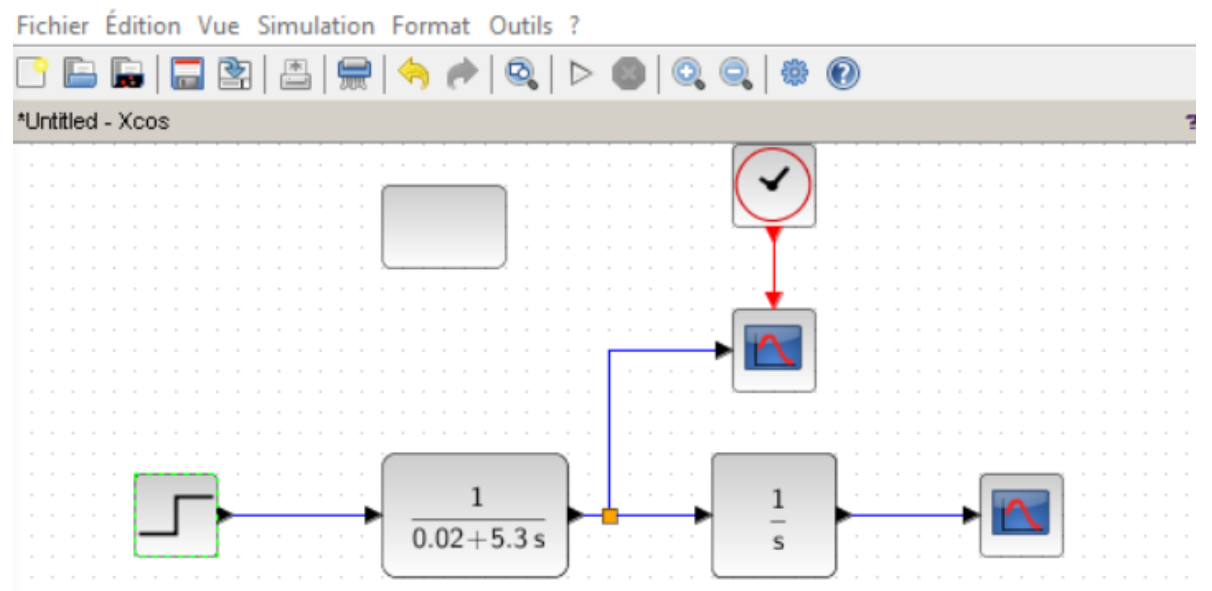

Figure 2. modélisation du contrôleur de vols du drone
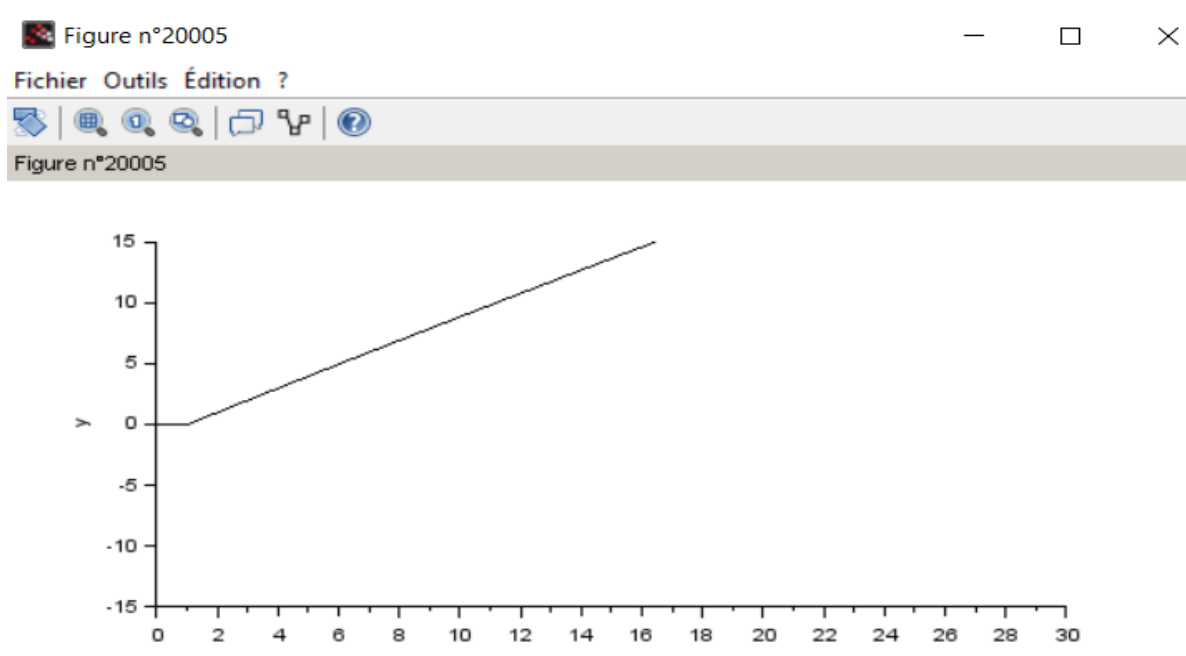

Figure 3. simulation avec valeurs aléatoire des $P, I, D$

Cette figure montre que le drone gagne d'altitude sans cesse cela signifie de point de vue réelle qu'il ne peut pas être stabilisé autour d'une altitude. On va donc essayer directement avec un PID, On est allée d'abord avec les valeurs mentionnées dans la littératures ; 

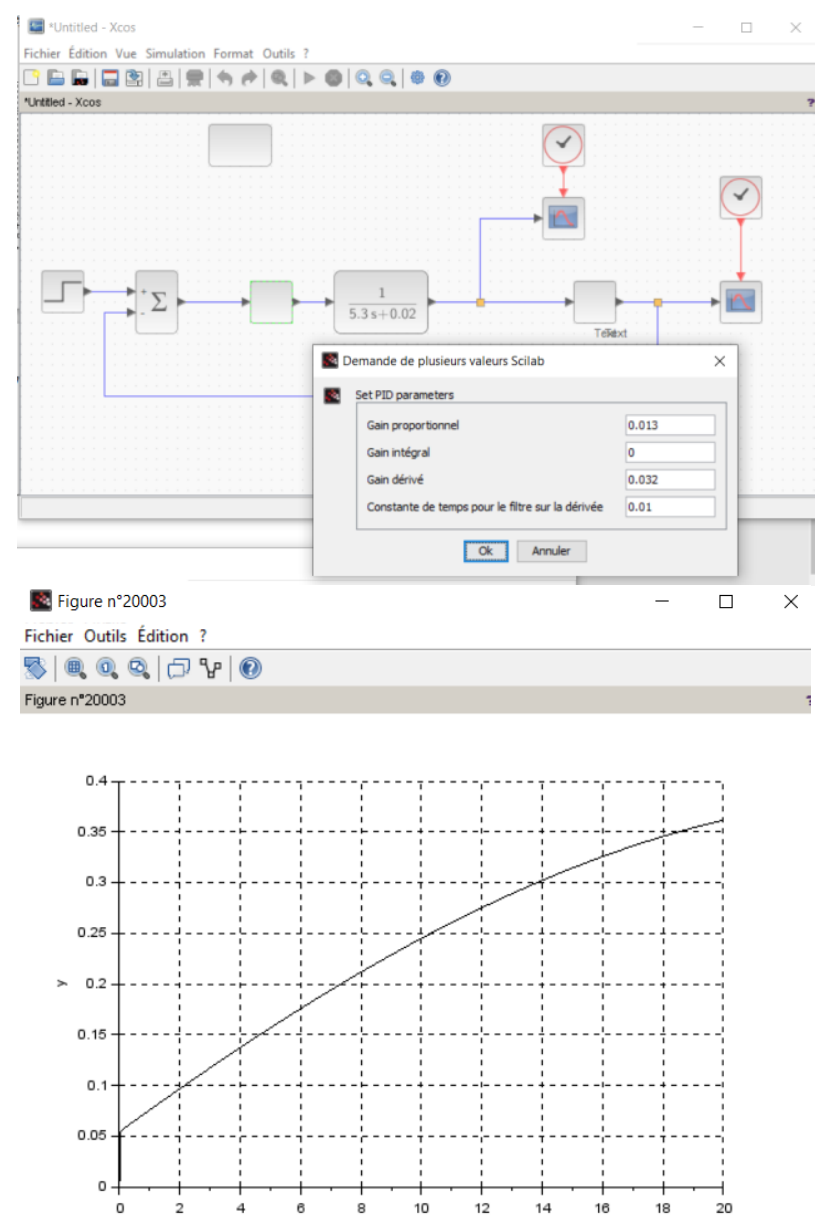

Figure 4. vitesse en fonction de l'altitude après un premier cycle d'optimisation

On remarque que la vitesse ne montre aucun critère de stabilité si on change la taille de la figure avant la simulation on peut voir que la vitesse ne trouve pas une valeur de stabilité.

Le PID parfait n'existe pas, tout est une question de compromis. Après un nombre d'essai pour limiter le choix des valeur de $\mathrm{P}$, I et $\mathrm{D}$ ( en prenant $\mathrm{I}=0$ dès le début du fait qu'on a un intégrateur dans la fonction de transfert introduit par le $1 / \mathrm{s}$ ). 

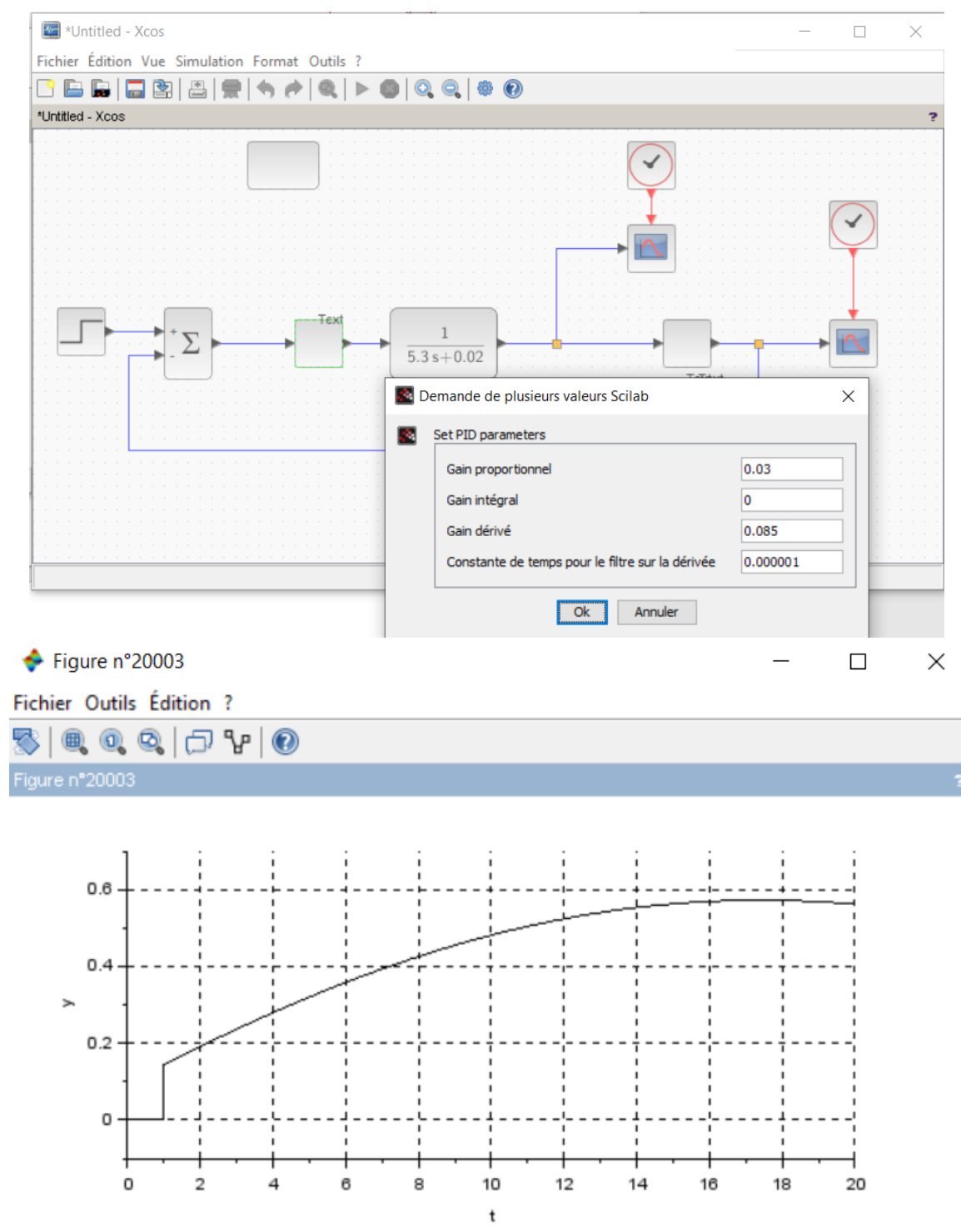

Figure 5. Vitesse stabilisée

On augmente le $\mathrm{P}$ jusqu'à avoir des vibration ou fluctuation de vitesse cela nous montre qu'on a atteint la valeur autour du qu'elle on peut stabiliser le drone.[4]

On augmente après le I on veut avoir des perturbation qui dure moins de temps, mais d'autre coté ses fluctuations vont être plus intense en effet c'est le rôle de l'intégrateur il va calculer l'air entre la ligne qui présente la stabilité de vitesse ( valeur constante ) et le tracé abouti après l'augmentation de la valeur de $\mathrm{P}$, puisque l'air entre l'échelon et le tracé est le plus important on va avoir une diminution principale de cette partie , ce qui signifie dans le cas réelle une diminution du temps de stabilisation. C'est donc le rôle du dérivateur qui va atténuer les fluctuations jusqu'au stabilisation de vitesse.

\section{Conclusion}

Avec optimisation des paramètres du contrôleurs de vol on a trouvé des valeurs qui ont assuré une certaine stabilité après une période de temps réduite. Ces travaux sont faits pour un vol vertical du drone et peuvent être réutiliser pour le vol stationnaire.

Des travaux supplémentaires vont être faites pour les autre phases de profil de mission, les contraintes de poids, vitesse de vols et conditions climatiques vont être prise en considération. 


\section{Référence}

[1] EL MAANI, Rabii, RADI, Bouchaïb, et EL HAMI, Abdelkhalak. Vibratory Reliability Analysis of an Aircraft's Wing via Fluid-Structure Interactions. Aerospace, 2017, vol. 4, no 3, p. 40.

[2] EL MAANI, R., RADI, B., et EL HAMI, A. Simulation numérique de l'écoulement autour d'une éolienne à axe horizontal. 2019.

[3] BELKADI, Adel, OULHADJ, Hamouche, TOUATI, Youcef, et al. On the robust PID adaptive controller for exoskeletons: A particle swarm optimization based approach. Applied Soft Computing, 2017, vol. 60, p. 87-100.

[4] KALKOUL, Youcef Islem, BERKANI, Thabet, et KHADRAOUI, Amar. Application Des Méthodes D’optimisation Pour La Commande D’un Drone. 2021. 